\title{
ShinyOmics: collaborative exploration of omics-data
}

\author{
Defne Surujon *iD and Tim van Opijnen ${ }^{*}$
}

\begin{abstract}
Background: Omics-profiling is a collection of increasingly prominent approaches that result in large-scale biological datasets, for instance capturing an organism's behavior and response in an environment. It can be daunting to manually analyze and interpret such large datasets without some programming experience. Additionally, with increasing amounts of data; management, storage and sharing challenges arise.

Results: Here, we present ShinyOmics, a web-based application that allows rapid collaborative exploration of omics-data. By using Tn-Seq, RNA-Seq, microarray and proteomics datasets from two human pathogens, we exemplify several conclusions that can be drawn from a rich dataset. We identify a protease and several chaperone proteins upregulated under aminoglycoside stress, show that antibiotics with the same mechanism of action trigger similar transcriptomic responses, point out the dissimilarity in different omics-profiles, and overlay the transcriptional response on a metabolic network.

Conclusions: ShinyOmics is easy to set up and customize, and can utilize user supplied metadata. It offers several visualization and comparison options that are designed to assist in novel hypothesis generation, as well as data management, online sharing and exploration. Moreover, ShinyOmics can be used as an interactive supplement accompanying research articles or presentations.
\end{abstract}

Keywords: Systems biology, Visualization, Transcriptomics, Functional genomics, Data integration

\section{Background}

Omics-profiling is becoming increasingly prevalent in many subfields in biology. For example, genome-wide transcriptomics have been used in studies of gene expression during embryonic stem cell differentiation, host-pathogen interactions, identification of biomarkers associated with antibiotic resistance and cancer disease progression [1-8]. Similarly, proteomic screens can identify proteins relevant for virulence, or cancer biomarkers [9-12]. Furthermore, phenotypic profiling using transposon insertion sequencing (Tn-Seq) in human pathogens has identified genes involved in colonization, infection, and intrinsic antibiotic resistance; and has been used in genetic interaction mapping [13-18].

Since genome-wide multi-omic profiling is paving the way to such varied and clinically relevant applications, considerable effort has gone into establishing analysis pipelines that process the resulting data. Tools such as

* Correspondence: surujon@bc.edu; vanopijn@bc.edu

Biology Department, Boston College, Chestnut Hill, MA 02467, USA
DESeq2 [19] and MAGenTA [20] are used for statistical analysis of differential gene expression and fitness changes respectively. However, the volume of the analyzed data can make interpretation and comprehensive evaluation non-trivial. Moreover, these tools often do not accommodate easy incorporation of metadata pertaining to genes and/or experimental conditions. This makes it time consuming and labor intensive to apply custom analysis protocols on each dataset, especially if the user has limited programming experience.

Existing tools for user-friendly data exploration and visualization include Stemformatics [21], Metascape [22], and mixOmics [23]. Stemformatics is an online portal that assembles gene expression data from stem cell datasets. While it provides an interactive visual interface, Stemformatics is tailored for stem cell research, and hosts a specific and focused dataset that does not expand to fields other than stem cell research. Metascape does allow users to supply their own datasets (often in the form of a gene list extracted from differential expression or other omics profiling data), and can merge information 
from public databases as well as perform functional enrichment and network analyses. The heavy dependence on well-curated annotation and information on public databases can be a limitation for researchers working with less well-characterized organisms, where these annotations may not be readily available; or available to the user but not yet made public. Moreover, even though the user can provide gene lists extracted from different omics screens, these analyses are performed independently. mixOmics is an $\mathrm{R}$ package that allows the user to interact with and analyze their own (potentially unpublished) data with less reliance on public databases, and consider multi-omics data simultaneously. It provides multiple pipelines focused on dimensionality reduction and feature selection, which can be extremely valuable in determining what signatures are associated with for instance disease outcome. However, if a researcher's interests are more specific, e.g. asking what expression changes are observed for a specific set of genes, a more customizable platform may be better suited.

To complement existing tools, we present ShinyOmics, a browser-based interface that allows for customizable visualizations of genome-wide profiling data, incorporating user-supplied metadata from genes and experimental conditions, and network connectedness of genes. It is straightforward to swap out the existing datasets loaded in ShinyOmics with user-generated custom data; e.g. standard output from DESeq2 can directly be incorporated. This feature of ShinyOmics also facilitates data management and sharing; for example, a lab can host a fully interactive instance of ShinyOmics with their own data making it accessible to collaborators across the world through a URL. This creates a convenient alternative over transferring and describing a large number of spreadsheets and data files between labs. Moreover, ShinyOmics can be deployed with new data obtained in a research project, as an interactive supplement that can be included in a manuscript submission, or academic presentation.

\section{Implementation}

ShinyOmics was developed in $\mathrm{R}$ version 3.4 .3 [24], using RStudio version 1.1.419 [25]. Running the app locally requires the packages ggplot2 [26] (v3.1.0), visNetwork [27] (v2.0.5), RColorBrewer [28] (v.1.1), igraph [29] (v1.2.2), heatmaply [30] (v.0.16.0), shinyHeatmaply [31] (v.0.1.0) and shiny [32] (v1.2.0).

An example of the app with data from [33-35] is available at [36]. The source code for the app and detailed usage notes can be accessed from [37]. Detailed usage notes are also provided in the aforementioned link.

There are three types of custom data that can be added; genome-wide profiling data, strain metadata, and network data. The main reference file for the app is "exptsheet.csv" under the "data" subdirectory. Any added experiment needs to be recorded in this file, with the corresponding profiling and metadata file locations specified. At minimum exptsheet.csv should have columns "Experiment", "Time", "Name", "DataFile", "Strain", and "MetadataFile". There can be as many additional columns as desired to record metadata of the experiments. For profiling data files, the standard output of DESeq2 can be directly transferred to the "data" directory. Alternatively, a file with at least the columns "Gene", "Value" (e.g. $\log 2$ fold change of expression), and "padj" can be provided. While the data source can be any organism or strain, eukaryotic datasets with tens of thousands of genes are likely to cause significant lag in the application loading. We therefore recommend, in the case of eukaryotic data, filtering the dataset (based on the number and quality of reads, or variability among replicates) and working with only a subset of a few thousand genes at most. There needs to be one metadata file per strain, and the minimum requirement for each metadata file is one column labeled "Gene". Each metadata file can have as many columns as desired, all selectors on the app will adjust accordingly. Finally, the networks should be specified as edge tables, with two columns: "source" and "target", and be named "[Name]_Edges.csv" in the "data/ networks/" subdirectory. The network statistics will be computed automatically.

When the app is first loaded in the browser, all data/ metadata files and the experiment sheet will be screened and validated for the requirements mentioned above. If the files provided do not fit these specifications, pop up error messages will indicate what caused the validation to fail, in which file(s), and the app will load with no data.

\section{Results}

We provide a version of ShinyOmics pre-loaded with multi-omic data from two human pathogens; Streptococcus pneumoniae and Mycobacterium tuberculosis. The $S$. pneumoniae dataset includes Tn-Seq and RNA-Seq data from two strains (TIGR4 and 19F) that were exposed to $1 x$ Minimum Inhibitory Concentration (MIC) of kanamycin (KAN), levofloxacin (LVX), rifampicin (RIF), vancomycin (VNC) and penicillin (PEN) for 2-4h [33]. Differential expression (DE) on the RNA-Seq data was evaluated as the fold change in transcript abundance comparing antibiotic conditions to a no-antibiotic control using DESeq2 [19]. Fitness change (dW) on the Tn-Seq data was evaluated comparing antibiotic to no-antibiotic conditions as described in [17]. The $M$. tuberculosis dataset includes microarray data [34] and proteomics data [35] under hypoxic conditions over a span of up to 20 days of culture in vitro. In its current configuration there are four panels that allow 
for different types of visualization: Single Experiment, Comparison of 2 Experiments, Comparison of All Experiments, and Network Visualization.

In ShinyOmics the first panel is designed to explore relationships between a value associated with all genes (e.g. DE, $\mathrm{dW}$, protein abundance) and any other user supplied metadata (Fig. 1). The metadata variables and their descriptions can be found in Additional file 1: Table S1. The user can include other genome-wide profile data (e.g. change in fitness, $\mathrm{dW})$ in the metadata fields, or as a separate experimental data file. In the Single Experiment panel, DE is plotted against the selected metadata type. For instance, in the preloaded dataset, one can answer whether there are significant DE changes appearing in a specific cellular function, by selecting "Tag1" (primary functional tag of the gene) from the dropdown menu labelled "Variable" (Fig. 1). The resulting scatter plot has each gene as a point, with the categorical variable "Tag1" on the $\mathrm{x}$-axis and DE on the $\mathrm{y}$-axis. The plot is faceted by timepoints, i.e. each timepoint in the selected experiment is a separate panel. The user can select which timepoints to display or hide using the checkboxes on the right. There are several visualization tuning options, such as changing the transparency of points, or in the case of categorical $\mathrm{x}$-axis variables, adding some noise (or "jitter") to the $\mathrm{x}$-coordinate of each point (such that individual points do not overlap) and/or superimposing a violin plot. It is also possible to display only a subset of genes by pasting a gene list in the text box ("Paste gene list"), subsetting the genes by a metadata variable ("Select genes by metadata variable"), or to select genes directly from the plot by dragging a rectangle to define a region of interest (or "brushing") the plot. The brushed genes will be displayed in the table below. Clicking anywhere on the plot will reset the brushing. In the example provided, it is possible to identify a set of genetic information processing genes that are upregulated drastically when $S$. pneumoniae is exposed to kanamycin (Fig. 1). Kanamycin, an aminoglycoside, is a protein synthesis inhibitor that triggers the incorporation of erroneous amino acids during protein synthesis, leading to an accumulation of misfolded proteins [38]. In S. pneumoniae TIGR4, the Clp protease ATP-binding subunit (SP_ 0338) is upregulated 256-fold (Fig. 1), indicating a response by this organism to alleviate the antibiotic stress through the destruction of misfolded proteins. This is accompanied by the simultaneous upregulation of chaperones dnaK and grpE (SP_0517 and SP_0516), whose function it is to repair denatured and misfolded proteins [39].



Fig. 1 Single Experiment panel of ShinyOmics. The tabs above allow the user to navigate to different panels. On the left, there is an experiment selector (where options are populated from the experiment sheet supplied by the user), a gene list selector (when empty, all genes are displayed), a variable selector, and several visualization customization options. Here, the T4 kanamycin ("T4_KAN") experiment is displayed as a scatterplot. Setting the $\mathrm{x}$-axis variable to "Tag1" splits genes by functional Tag. 4 genes are brushed at timepoint 240 (blue rectangle), whose identity and metadata are displayed in the table (bottom) 
The Compare 2 Experiments panel allows for quick pairwise comparisons of experiments (Fig. 2). Here, one can plot the DE of one experiment against another, for the timepoints that are in common in both experiments. There is a selector for the color of the points (e.g. one can color each gene by functional category, or any other metadata feature). The plot is brushable, similar to the Single Experiment panel. As an example, the DE of two antibiotics are compared in Fig. 2. Vancomycin and penicillin are both cell wall synthesis inhibitors, and the transcriptomic changes in response to these antibiotics appear highly correlated, especially in the later timepoints (Fig. 2). This global similarity in transcriptional profiles is unique to the PEN-VNC pair, and is not observed when comparing antibiotics of different classes. In contrast, at 90-min a group of genes are brushed (SP 0044-SP_0054, Fig. 2) belonging to the category "Nucleotide metabolism" that turn out to be downregulated across most of the tested antibiotics, including the RNA synthesis inhibitor Rifampicin, and the DNA synthesis inhibitor Levofloxacin. This set of genes are part of the purine biosynthesis pathway, and their downregulation might point to a common antibiotic response in S. pneumoniae TIGR4.

It is also possible to see whether different systems under the same condition harbor similar responses using the Compare 2 Experiments panel. Comparison of TnSeq and RNA-Seq data from S. pneumoniae antibiotic experiments and a comparison of microarray and proteomic data from $M$. tuberculosis shows a lack of similarity in the responses in the different screens (Additional file 1: Figure S1). This is in accordance with previous findings that systems-level data are often quite distinct, and different systems should not be taken as substitutes of one another, but rather complementary parts of the organism as a whole $[18,40]$.

To identify general patterns across many experimental conditions, the Compare All Experiments panel can be used (Fig. 3). On the left of this panel, a heatmap shows all genes across all conditions, with optional dendrograms showing hierarchical clustering. The heatmap on the bottom is interactive, and shows only a userspecified set of genes, and conditions. On the right side of the panel, principal component analysis (PCA) results are visualized. The first scatter plot shows all experiments on any combination of the top 10 principal components. The user can select which components to plot, and a metadata variable to color the points by (e.g. in order to see whether the experiments are separated by antibiotic, one can select "AB" as the color variable in the pre-loaded dataset). For instance, Fig. 3 shows clear separation of Rifampicin from the other 4 antibiotics.

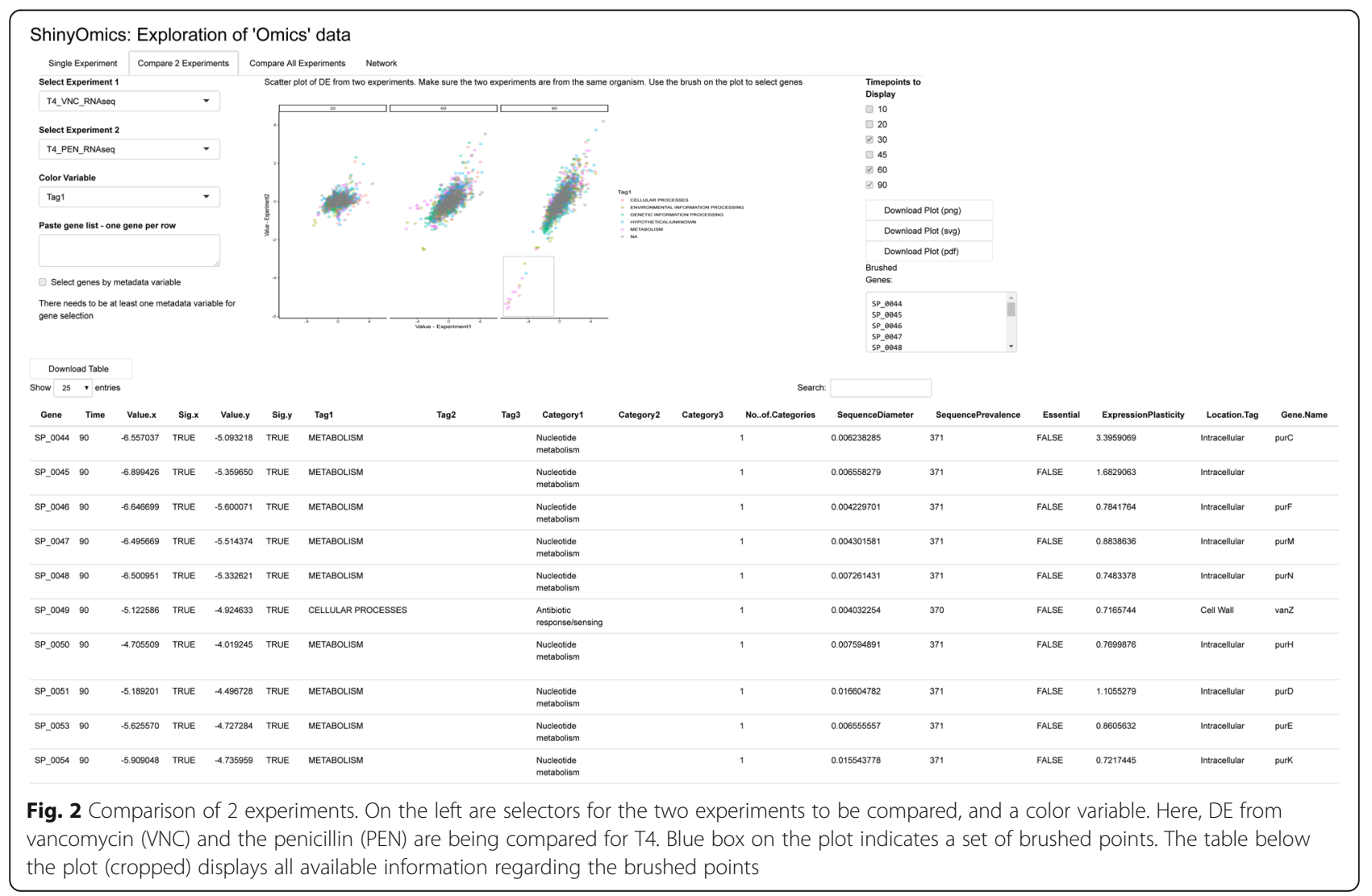




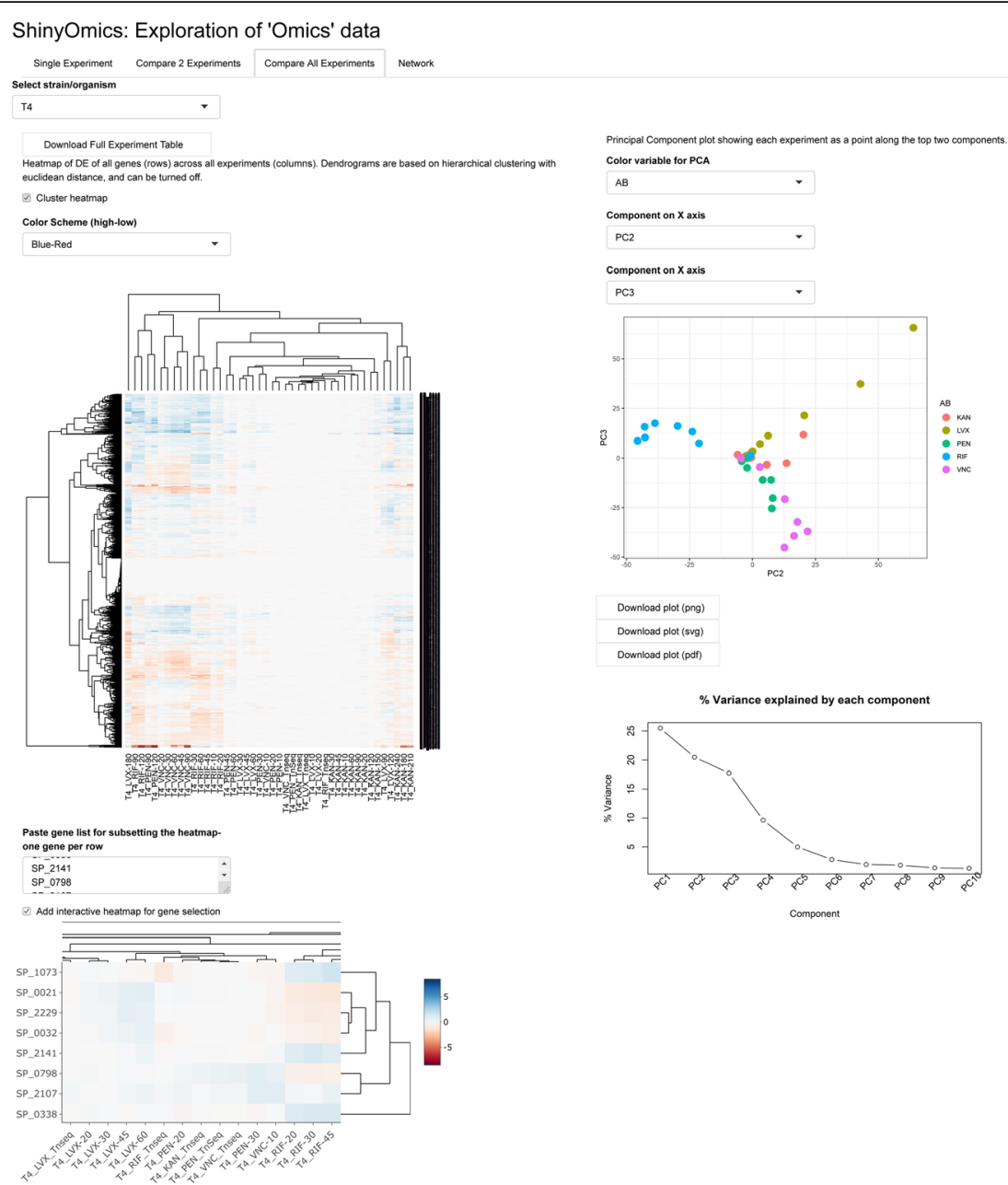

Fig. 3 Comparison of all experiments from the same strain. The heatmap shows DE of all experiments included in the experiment sheet for a specific strain (T4: TIGR4). The dendrogram on the heatmap and the PCA (colored by antibiotic) shows that the RNA synthesis inhibitor rifampicin (RIF) is most dissimilar to other antibiotics. AB: antibiotic. KAN: Kanamycin. LVX: Levofloxacin. VNC: Vancomycin. PEN: Penicillin

Rifampicin, being an RNA synthesis inhibitor, elicits the most dramatic changes in expression out of the 5 antibiotics included. The last plot shows the percent variance explained by each principal component. The informative components will be those that explain more of the variance in the data. A common way of selecting important components is to look for an 'elbow' in the last plot (i.e. a relatively clear point on a line where the slope changes drastically) and consider the components before the elbow [41].

In order to evaluate whether genes with for instance significant DE (DEGs) or dW are related to one another in a network context, the last panel (Network) allows visualization of a user supplied network of genes. Common types of biologically meaningful networks include proteinprotein interaction [42], transcription regulatory [43] metabolic [44] and genetic interaction [45] networks. Depending on the organism, these networks can be manually curated, inferred bioinformatically [46-48], or might already be experimentally mapped out. The preloaded metabolic networks were generated by Jensen et al. [18]. It is also important to keep in mind what kind of network is being used, in order to draw meaningful conclusions from the network analysis. For example, all DEGs localizing on a certain part of the transcription regulatory network may be a result of the DEGs belonging to the same regulon. However, the same phenomenon on a metabolic network may mean a specific metabolic pathway is being activated, which would imply a functional relationship between DEGs. The panel allows the user to select the experiment, timepoint and network, leading to DEGs marked on the network as red and blue nodes for up- and down-regulation respectively. On the example metabolic network of S. pneumoniae 19F (initially generated in [18]), the 120-min VNC response is overlaid (Fig. 4). It is possible to pick out numerous groups of interconnected genes that are up- or down-regulated together, although there are also examples of upregulated genes being adjacent to downregulated or non-DE genes. 


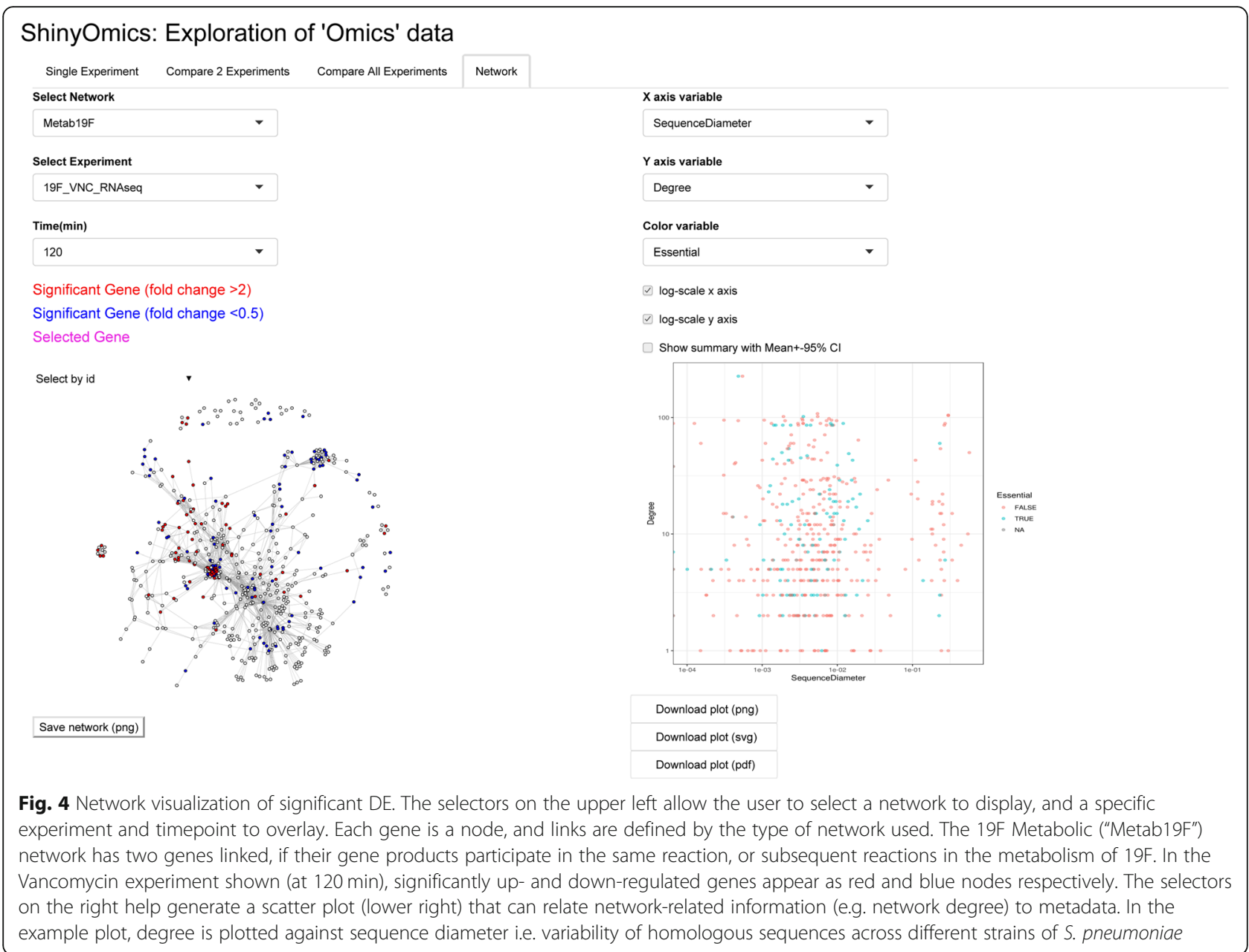

On the left, the network itself will be visualized in an interactive plot that allows zooming, selecting and dragging of nodes. On the right, a set of selectors allow for a custom scatter plot to be made, relating network characteristics of nodes (e.g. degree) to DE or any other metadata supplied by the user. As an example, network degree is plotted against sequence diameter (how variable the sequence is across multiple strains of $S$. pneumoniae), and genes are colored by whether or not they are essential in 19F (Fig. 4), showing a lack of relationship between these variables. Similar to scatter plots in the other panels, this plot is also brushable, and brushed points are displayed in the table below.

\section{Conclusions}

While genome-wide profiling can be incredibly valuable in a variety of applications, initial exploratory analysis of large datasets can be a daunting task. For instance, enumerating the DE of each gene with tools such as DESeq2 is a necessary but insufficient step in such analyses. ShinyOmics is a simple platform for facilitating initial exploratory analysis of omic-profiling data and hypothesis generating. The emphasis on relating genome-wide profiling to custom, user supplied metadata enables the user to make functional associations between any set of features of genes. Moreover, ShinyOmics serves as a convenient data management and sharing tool. Deploying an instance of ShinyOmics with data from a new study results in an interactive supplement for research articles or presentations. For example, a modified version of ShinyOmics accompanying a manuscript with the full antibiotic response dataset from [33] can be found at [49].

\section{Availability and requirements}

Project name: ShinyOmics

Project home page: https://github.com/dsurujon/ ShinyOmics

Operating system: Platform independent

Programming language: $\mathrm{R}$ (v.3.4.3)

Other requirements: ggplot2 v.3.2.0, visNetwork v.2.0.7, RColorBrewer v.1.1, igraph v.1.2.4, heatmaply v.0.16.0, shinyHeatmaply v.0.1.0, shiny v.1.3.2

License: Affero GPLv3

Any restrictions to use by non-academics: None 


\section{Supplementary information}

Supplementary information accompanies this paper at https://doi.org/10. 1186/s12859-020-3360-x

Additional file 1: Table S1. Metadata variables included in the example application, and their descriptions. Figure S1. Lack of overlap between different omics data. A. For the TIGR4 KAN experiment, RNA-Seq (Experiment 1) is plotted against Tn-seq (Experiment 2). B. For the M. tuberculosis hypoxia experiment, microarray data (Experiment1) is plotted against proteomics data (Experiment 2).

\section{Abbreviations}

DE: Differential expression; DEG: Differentially expressed gene; dW: Difference in fitness; KAN: Kanamycin; LVX: Levofloxacin; PEN: Penicillin; RIF: Rifampicin; VNC: Vancomycin

\section{Acknowledgements}

The authors would like to thank the members of the van Opijnen lab, especially Juan C. Ortiz-Marquez and Indu Warrier who have used ShinyOmics and provided helpful suggestions in improving it; and Anthony Schreiner for his help in setting up the shiny server.

\section{Authors' contributions}

DS developed the application and drafted the paper. DS and TVO read and approved the final manuscript.

\section{Funding}

This work was supported by the National Institutes of Health of USA (R01 Al124302, U01 Al110724, R01 GM115931) awarded to TVO. The funding body did not have a role in the design of this project; data collection, analysis and interpretation; and writing of this manuscript.

\section{Availability of data and materials}

The example dataset, user guide and a code for ShinyOmics can be found in the github ShinyOmics repository, https://github.com/dsurujon/ShinyOmics, or as a capsule on CodeOcean [50]

An example of the application can be accessed at the URL http:// bioinformatics.bc.edu/shiny/ShinyOmics/.

\section{Ethics approval and consent to participate}

Not applicable.

\section{Consent for publication}

Not applicable.

\section{Competing interests}

The authors declare that they have no competing interests.

Received: 3 July 2019 Accepted: 10 January 2020

Published online: 17 January 2020

\section{References}

1. Cloonan N, Forrest ARR, Kolle G, Gardiner BBA, Faulkner GJ, Brown MK, et al. Stem cell transcriptome profiling via massive-scale mRNA sequencing. Nat Methods. 2008;5(7):613-9.

2. Westermann AJ, Förstner KU, Amman F, Barquist L, Chao Y, Schulte LN, et al. Dual RNA-seq unveils noncoding RNA functions in host-pathogen interactions. Nature. 2016:529(7587):496-501.

3. Aprianto R, Slager J, Holsappel S, Veening J-W. Time-resolved dual RNA-seq reveals extensive rewiring of lung epithelial and pneumococcal transcriptomes during early infection. Genome Biol. 2016;17:198.

4. Barczak AK, Gomez JE, Kaufmann BB, Hinson ER, Cosimi L, Borowsky ML, et al. RNA signatures allow rapid identification of pathogens and antibiotic susceptibilities. Proc Natl Acad Sci U S A. 2012;109(16):6217-22.

5. Khaledi A, Schniederjans M, Pohl S, Rainer R, Bodenhofer U, Xia B, et al. Transcriptome profiling of antimicrobial resistance in Pseudomonas aeruginosa. Antimicrob Agents Chemother. 2016;60(8):4722-33.

6. van't Veer $L$, Dai H, van de Vijver MJ, He YD, Hart AAM, Mao M, et al. Gene expression profiling predicts clinical outcome of breast cancer. Nature. 2002; 415(6871):530
7. Ozsolak F, Milos PM. RNA sequencing: advances, challenges and opportunities. Nat Rev Genet. 2011;12(2):87-98.

8. Lowe R, Shirley N, Bleackley M, Dolan S, Shafee T. Transcriptomics technologies. PLoS Comput Biol. 2017:13(5):e1005457.

9. Yimer SA, Birhanu AG, Kalayou S, Riaz T, Zegeye ED, Beyene GT, et al. Comparative proteomic analysis of Mycobacterium tuberculosis lineage 7 and lineage 4 strains reveals differentially abundant proteins linked to slow growth and virulence. Front Microbiol. 2017:8:795.

10. Adam B-L, Qu Y, Davis JW, Ward MD, Clements MA, Cazares LH, et al. Serum protein fingerprinting coupled with a pattern-matching algorithm distinguishes prostate Cancer from benign prostate hyperplasia and healthy men. Cancer Res. 2002;62(13):3609-14.

11. Bantscheff M, Lemeer S, Savitski MM, Kuster B. Quantitative mass spectrometry in proteomics: critical review update from 2007 to the present. Anal Bioanal Chem. 2012:404(4):939-65.

12. Anjo SI, Santa C, Manadas B. SWATH-MS as a tool for biomarker discovery: from basic research to clinical applications. Proteomics. 2017;17(3-4):1600278.

13. van Opijnen T, Camilli A. Transposon insertion sequencing: a new tool for systems-level analysis of microorganisms. Nat Rev Microbiol. 2013;11(7):435-42.

14. van Opijnen T, Bodi KL, Camilli A. Tn-seq: high-throughput parallel sequencing for fitness and genetic interaction studies in microorganisms. Nat Methods. 2009;6(10):767-72.

15. van Opijnen T, Dedrick S, Bento J. Strain dependent genetic networks for antibiotic-sensitivity in a bacterial pathogen with a large pan-genome. PLoS Pathog. 2016;12(9):e1005869.

16. Gallagher LA, Shendure J, Manoil C. Genome-Scale Identification of Resistance Functions in Pseudomonas aeruginosa Using Tn-seq. mBio. 2011:2(1):e00315-0.

17. van Opijnen T, Camilli A. A fine scale phenotype-genotype virulence map of a bacterial pathogen. Genome Res. 2012;22(12):2541-51.

18. Jensen PA, Zhu Z, van Opijnen T. Antibiotics disrupt coordination between transcriptional and phenotypic stress responses in pathogenic bacteria. Cell Rep. 2017;20(7):1705-16.

19. Love Ml, Huber W, Anders S. Moderated estimation of fold change and dispersion for RNA-seq data with DESeq2. Genome Biol. 2014;15(12):550.

20. McCoy KM, Antonio ML, van Opijnen T. MAGenTA: a galaxy implemented tool for complete Tn-Seq analysis and data visualization. Bioinformatics. 2017;33(17):2781-3.

21. Choi J, Pacheco CM, Mosbergen R, Korn O, Chen T, Nagpal I, et al. Stemformatics: visualize and download curated stem cell data. Nucleic Acids Res. 2019:47(D1):D841-6.

22. Zhou Y, Zhou B, Pache L, Chang M, Khodabakhshi AH, Tanaseichuk O, et al. Metascape provides a biologist-oriented resource for the analysis of systems-level datasets. Nat Commun. 2019;10(1):1-10.

23. Rohart F, Gautier B, Singh A, Cao K-AL. mixOmics: An R package for 'omics feature selection and multiple data integration. PLOS Comput Biol. 2017; 13(11):e1005752.

24. R Core Team. R: A Language and Environment for Statistical Computing. R Foundation for Statistical Computing. 2017. https://www.R-project.org/. Accessed 28 Oct 2019

25. RStudio Team. RStudio: integrated development environment for R: RStudio, Inc:; 2015. http://www.rstudio.com/. Accessed 28 Oct 2019

26. Wickham H. ggplot2: Elegant Graphics for Data Analysis: Springer-Verlag; 2016. Available from: https://ggplot2.tidyverse.org. Accessed 28 Oct 2019

27. Almende BV, Thieurmel B, Robert T. visNetwork: Network Visualization using "vis.js" Library. 2019. Available from: https://CRAN.R-project.org/package= visNetwork Accessed 28 Oct 2019.

28. Neuwirth E. RColorBrewer: ColorBrewer palettes. 2014. https://CRAN.Rproject.org/package=RColorBrewer. Accessed 28 Oct 2019.

29. Csardi G, Nepusz T. The igraph software package for complex network research. InterJournal. 2006; Complex Systems:1695.

30. Galili T, O'Callaghan A, Sidi J, Sievert C. heatmaply: an R package for creating interactive cluster heatmaps for online publishing. Bioinformatics. 2017;34(9):1600-2

31. Sidi J, Galili T. shinyHeatmaply: Deploy 'heatmaply' using 'shiny'. 2017. https://CRAN.R-project.org/package=shinyHeatmaply. Accessed 28 Oct 2019.

32. Chang W, Cheng J, Allaire JJ, Xie Y, McPherson J, RStudio, et al. shiny: Web Application Framework for R. 2019. https://CRAN.R-project.org/package= shiny. Accessed 28 Oct 2019.

33. Zhu Z, Surujon D, Ortiz-Marquez JC, Wood SJ, Huo W, Isberg RR, et al. Entropy of a bacterial stress response is a generalizable predictor for fitness and antibiotic sensitivity. bioRxiv. 2019;22:813709. 
34. Galagan JE, Minch K, Peterson M, Lyubetskaya A, Azizi E, Sweet L, et al. The Mycobacterium tuberculosis regulatory network and hypoxia. Nature. 2013; 499(7457):178-83.

35. Schubert OT, Ludwig C, Kogadeeva M, Zimmermann M, Rosenberger G, Gengenbacher $\mathrm{M}$, et al. Absolute proteome composition and dynamics during dormancy and resuscitation of Mycobacterium tuberculosis. Cell Host Microbe. 2015;18(1):96-108

36. ShinyOmics: Exploration of "Omics" data. 2019. http://bioinformatics.bc.edu/ shiny/ShinyOmics/. Accessed 29 Oct 2019.

37. ShinyOmics User Guide. 2019. https://github.com/dsurujon/ShinyOmics. Accessed 29 Oct 2019

38. Goltermann L, Good L, Bentin T. Chaperonins fight aminoglycoside-induced protein Misfolding and promote short-term tolerance in Escherichia coli. J Biol Chem. 2013;288(15):10483-9.

39. Schröder H, Langer T, Hartl FU, Bukau B. DnaK, DnaJ and GrpE form a cellular chaperone machinery capable of repairing heat-induced protein damage. EMBO J. 1993;12(11):4137-44.

40. Ghazalpour A, Bennett B, Petyuk VA, Orozco L, Hagopian R, Mungrue IN, et al. Comparative analysis of proteome and Transcriptome variation in mouse. PLoS Genet. 2011;7(6):e1001393.

41. Cangelosi R, Goriely A. Component retention in principal component analysis with application to CDNA microarray data. Biol Direct. 2007;2(1):2.

42. Schwikowski B, Uetz P, Fields S. A network of protein-protein interactions in yeast. Nat Biotechnol. 2000;18(12):1257-61.

43. Fang X, Sastry A, Mih N, Kim D, Tan J, Yurkovich JT, et al. Global transcriptional regulatory network for Escherichia coli robustly connects gene expression to transcription factor activities. PNAS. 2017;114(38):10286-91.

44. Jeong H, Oltvai ZN, Barabási A-L. Prediction of protein essentiality based on genomic data. Complexus. 2003;1(1):19-28.

45. Costanzo M, VanderSluis B, Koch EN, Baryshnikova A, Pons C, Tan G, et al. A global genetic interaction network maps a wiring diagram of cellular function. Science. 2016;353(6306):aaf1420.

46. Huang L, Liao L, Wu CH. Protein-protein interaction network inference from multiple kernels with optimization based on random walk by linear programming. In: 2015 IEEE International Conference on Bioinformatics and Biomedicine (BIBM); 2015. p. 201-7.

47. Skwark MJ, Croucher NJ, Puranen S, Chewapreecha C, Pesonen M, Xu YY, et al. Interacting networks of resistance, virulence and core machinery genes identified by genome-wide epistasis analysis. PLoS Genet. 2017:13(2):e1006508.

48. Zhang B, Horvath S. A general framework for weighted gene co-expression network analysis. Stat Appl Genet Mol Biol. 2005;4:Article17.

49. Streptococcus pneumoniae antibiotic stress response. 2019. http://bioinformatics. bc.edu/shiny/ABX. Accessed 15 Oct 2019.

50. Surujon D, van Opijnen T. ShinyOmics: CodeOcean; 2019. https://doi.org/10. 24433/CO.2062023.v1. Accessed 28 Oct 2019

\section{Publisher's Note}

Springer Nature remains neutral with regard to jurisdictional claims in published maps and institutional affiliations.

Ready to submit your research? Choose BMC and benefit from:

- fast, convenient online submission

- thorough peer review by experienced researchers in your field

- rapid publication on acceptance

- support for research data, including large and complex data types

- gold Open Access which fosters wider collaboration and increased citations

- maximum visibility for your research: over $100 \mathrm{M}$ website views per year

At BMC, research is always in progress.

Learn more biomedcentral.com/submissions 